\title{
The Inhibition of H1N1 influenza virus-induced apoptosis by functionalized Selenium nanoparticles with $\beta$-Thujaplicin through ROS-mediated P53 and AKT signaling pathways
}

\section{Changbing Wang}

Guangzhou Women and Children's Medical Center, Guangzhou Medical University

\section{Mingqi Zhao}

Center Laboratory,Guangzhou Women and Children's Medical Center, Guangzhou Medical University Zhengfang Lin

Center Laboratory,Guangzhou Women and Children's Medical Center, Guangzhou Medical University Min Guo

Center Laboratory,Guangzhou Women and Children's Medical Center, Guangzhou Medical University Tiantian Xu

Center Laboratory,Guangzhou Women and Children's Medical Center, Guangzhou Medical University

\section{Ying Tang}

Center Laboratory,Guangzhou Women and Children's Medical Center,Guangzhou Medical University

\section{Yi Chen}

Center Laboratory,Guangzhou Women and Children's Medical Center,Guangzhou Medical University

\section{Liang Hua}

Center Laboratory,Guangzhou Women and Children's Medical Center,Guangzhou Medical University

\section{Jiayu Zhong}

Center Laboratory,Guangzhou Women and Children's Medical Center,Guangzhou Medical University

\section{Tao Lin}

Center Laboratory,Guangzhou Women and Children's Medical Center,Guangzhou Medical University

\section{Guangwan Lian}

Center Laboratory,Guangzhou Women and Children's Medical Center, Guangzhou Medical University Huanhui Chen

Center Laboratory,Guangzhou Women and Children's Medical Center,Guangzhou Medical University Bing Zhu

Center Laboratory,Guangzhou Women and Children's Medical Center, Guangzhou Medical University Yinghua Li ( $\nabla$ hualei_1314@hotmail.com )

Center Laboratory,Guangzhou Women and Children's Medical Center, Guangzhou Medical University https://orcid.org/0000-0002-6271-8283 


\section{Nano Express}

Keywords: Selenium nanoparticles, $\beta$-Thujaplicin, H1N1 influenza virus, Apoptosis

Posted Date: March 4th, 2020

DOl: https://doi.org/10.21203/rs.3.rs-15986/v1

License: (c) (i) This work is licensed under a Creative Commons Attribution 4.0 International License. Read Full License 


\section{Abstract}

$\beta$-Thujaplicin possess a variety of biological activities. The use of modified biological nanoparticles (NPs) to develop novel anti-influenza drugs has increased in recent years. Selenium nanoparticles (SeNPs) with antiviral has attracted increasing attention for biomedical intervention. Functionalized SeNPs by $\beta$-Thujaplicin (Se@TP) surface modified with superior antiviral were synthesized in this study. $\beta$-Thujaplicin decoration of SeNPs obviously inhibited H1N1 infection and were less toxicity. Se@TP could inhibit H1N1 from infecting Madin Darby Canine Kidney (MDCK) cells and block chromatin condensation and DNA fragmentation. Se@TP obviously prevented MDCK cells from generating reactive oxygen species (ROS). Furthermore, Se@TP prevent lung injury in H1N1 infected mice through eosin staining and hematoxylin in vivo. Additionally, when treated with Se@TP, the DNA damage of lung tissues reduced substantially by TUNEL-DAPI test. Mechanistic investigation revealed that Se@TP inhibited H1N1 influenza virus from infecting MDCK cells through induction of apoptosis via suppression AKT and p53 signaling pathways through Immunohistochemical assay. Our results suggest that $\beta$ Thujaplicin modified SeNPs as carriers is an efficient way to achieve antiviral pharmaceutical candidate for H1N1 influenza.

\section{Introduction}

As a segmented RNA virus, Influenza virus affects millions of people and is still a serious contagious pathogen in seasonal epidemics. ${ }^{1,2} \mathrm{H} 1 \mathrm{~N} 1$ influenza virus is a highly infections respiratory disease which belongs to influenza A type viruses. ${ }^{3}$ H1N1 Influenza virus was discovered and identified in US and Mexico in 2009 which infected more than 8768 deaths in 207 countries. ${ }^{4}$ Due to the arising mutation of antigenic shifts and the genome in different species, influenza virus may emerge a novel influenza among humans in the future. ${ }^{5}$ The influenza infection cycle included three steps: First, the influenza viruses attach to the host cell surface receptor and fuse with the endosomal membrane. Second, uncoating of nucleocapsid and multiplication of the genetic material occurs. Finally, the influenza protein and new viron is expressed and released. ${ }^{6,7}$ On the surface of influenza virus, there are two important glycoprotein: Hemagglutinin (HA) and Neuraminidase (NA) ${ }^{8}$ In the early stage of viral infection, HA combined sialic acid-containing receptors on host cells and mediated the entry and fusion of virus. ${ }^{9,10}$ when mature viruses separate from host cell surface, NA plays an important role in assisting virus cleave the linkage between sialic acid and Hemagglutinin. ${ }^{11}$ Although, the conventional way to restrain the spread of influenza infections is vaccination. But, the restraining the spread of influenza infections is the long period between the rapid virus evolution and vaccine development. ${ }^{12}$

$\beta$-Thujaplicin (2-hydroxy-4-isopropyl-2,4,6-cyclohepentatrieneone) is an antimicrobial tropolone derived from geranyl pyrophosphate (GPP) and monoterpene intermedicate. ${ }^{13,14} \beta$-Thujaplicin have been reported to possess antibacterial and antifungal activities. However, anti-influenza virus effects of $\beta$-Thujaplicin remain unclear. ${ }^{15,16}$ The nanotechnology provided a new prospect to solve these problems, and the new antiviral nanodrug should effectively inhibit viral infection with fewer cytotoxicity. ${ }^{17-19}$ Gold 
nanoparticles conjugated to consensus M2e peptide against influenza A viruses was reported by Tao et

al. ${ }^{20}$ Different sizes of polyvalent nanoparticles inhibited virus was reported by Vonnemann et al. ${ }^{21}$ Wang et al reported that $\mathrm{SiO}_{2} @ \mathrm{LDH}$ nanoparticles enhance the response of hepatitis $\mathrm{B}$ virus DNA vaccine. ${ }^{22}$ Shen et al reported efficient Encapsulation of $\mathrm{Fe}_{3} \mathrm{O}_{4}$ Nanopartilces into genetically engineered Hepatitis $\mathrm{B}$ core virus-like particles. ${ }^{23}$ Zhang et al reported silver nanoparticle treatment ameliorates biliary atresia syndrome in rhesus rotavirus inoculated mice. ${ }^{24}$ among of them, selenium nanoparticles (SeNPs) attract much attention due to the unique antimicrobial activities. ${ }^{25}$ Selenium is integral component of several selenoproteins which control several crucial biological processes. ${ }^{26,27}$ The deficiency of selenium could enhance the susceptibility to infections including respiratory virus infections. ${ }^{28}$ Therefore, the aim of the present study was to exposure novel functionalized selenium nanoparticles which can inhibit the infection of H1N1 virus. We hypothesized that $\beta$-Thujaplicin modified SeNPs (Se@TP) have excellent antiviral activity against $\mathrm{H} 1 \mathrm{~N} 1$ virus. Though several research groups have described the antimicrobial effects of SeNPs, the antiviral mechanisms is still unclear. This study was to verify how $\beta$-Thujaplicin modified SeNPs to inhibit H1N1 influenza virus in vitro and in vivo.

\section{Materials And Methods}

\section{Materials}

$\mathrm{Na}_{2} \mathrm{SeO}_{3}$, Vitamin $\mathrm{C}, \beta$-Thujaplicin, propidium iodide, 6 -coumarin were provided from Sigma. Fetal bovine serum (FBS) and Dulbecco's modified eagle medium (DMEM) and were purchased from Gibco. lyso tracker, Thiazolyl blue tetrazolium bromide (MTT) were from Sigma. phosphorylated p53 (p-p53), Phosphorylated AKT ( $\mathrm{p}-\mathrm{AKT}$ ) and $\beta$-actin antibodies were purchased from Cell Signaling Technology (CST). Madin darby canine kidney cells (MDCK) were obtained from American Type Culture Collection (ATCC® CCL-34TM). H1N1 influenza virus was provided by Guangzhou Women and Children's Medical Center, Guangzhou Medical University. Mice were obtained from the Guangdong Medical Laboratory Animal Center (Guangdong, China). All animal procedures were performed according to the guidelines of Guangdong Medical Laboratory Animal Center.

\section{Preparation and Characterization of Se@TP}

$\beta$-Thujaplicin modified Selenium nanoparticles were prepared as follow: $0.25 \mathrm{ml}$ stock solution of $\mathrm{Na}_{2} \mathrm{SeO}_{3}(0.1 \mathrm{M})$ was gradually added into $2 \mathrm{ml}$ stock solution $(50 \mathrm{mM})$ of Vitamin C. Then, $10 \mu \mathrm{l}$ $40 \mathrm{mg} / \mathrm{ml}$ of $\beta$-Thujaplicin was added into the selenium nanoparticles solution. The excess $\beta$-Thujaplicin, Vitamin and $\mathrm{Na}_{2} \mathrm{SeO}_{3}$ were removed by dialysis for overnight. Se@TP nanoparticles were sonicated and then filtered through $0.2 \mu \mathrm{m}$ pore size. The concentration of SeNPs and TP was measured by ICP-AES. The morphology and elemental composition of Se@TP nanoparticles were characterized by Transmission Electron Microscopy (TEM, H-7650) and EDX (EX-250 system, Horiba). Malvern Zetasizer Software was used to monitor the zeta potential and size distribution of Se@TP. 


\section{Determination of cell viability}

The cytotoxic of Se@TP nanoparticles was performed as previously reported. ${ }^{29,30}$ First, H1N1 influenza virus was added to MDCK cells for $2 \mathrm{~h}$, and then the indicated concentrations of $\beta$-Thujaplicin with or without SeNPs were added to MDCK cells for $24 \mathrm{~h}$. Then, MTT $(20 \mu \mathrm{l} /$ well) was added for $5 \mathrm{~h}$. The formazan crystals were recorded at the absorbance of $570 \mathrm{~nm}$.

\section{Intracellular localization of Se@TP}

MDCK cells were treated with lyso tracker for 60 min as previous reported. ${ }^{31}$ DAPI and coumarin-6 labelled Se@TP were added for various periods of time incubation. Fluorescence microscope was used to obtain and analysis the image.

\section{Caspase-3 activity and TUNEL-DAPI co-staining assay}

The caspase- 3 activity with the wavelengths at $380 \mathrm{~nm}$ (excitation) and $460 \mathrm{~nm}$ (emission) was detected as previously described. ${ }^{32}$ DNA fragmentation was examined with fluorescence staining by the TUNEL apoptosis detection kit. ${ }^{33}$ MDCK cells were confirmed with TUNEL for $1 \mathrm{~h}$ and incubated of DAPI for 15 min at $37^{\circ} \mathrm{C}$ for nuclear staining.

\section{Thin sections of MDCK cells and ROS generation}

TEM analysis of MDCK cells were detected as previous described. ${ }^{34}$ After incubation with H1N1 influenza virus for 2 h, MDCK cells were washed with PBS and incubated with Se@TP. Se@TP-treated MDCK cells induced ROS accumulation was detected as previously described. ${ }^{35}$ The ROS generation was monitored through the fluorescence intensity (excitation $500 \mathrm{~nm}$ and emission $529 \mathrm{~nm}$ ).

\section{Animals infection and treatment}

Fifteen female BALB/c mice (aged 4-6 weeks) were randomly divided into five groups as follow: control group, H1N1 virus, virus + TP, virus + SeNPs, virus + Se@TP. All mice were anesthetized with 10\% chloral hydrate at a dose of $3 \mu \mathrm{l} / \mathrm{g}$. Then the control group was treated with $20 \mu \mathrm{l}$ physiological saline by nasal dripping while the other four groups with $20 \mu \mathrm{l} \mathrm{H1N1}$ virus by nasal dripping as well. $24 \mathrm{~h}$ later, TP, SeNPs and Se@TP were administered to anesthetized mice respectively via intranasal absorption every $24 \mathrm{~h}$ thereafter for a total of three times. The lungs were extracted, affused with physiological saline and fixed in paraformaldehyde before hematoxylin and eosin (HE) staining, tunel test and immunohistochemistry staining. All mice experiments were approved and guided by the Ethics Committee of Guangzhou Medical University.

\section{Statistical analysis}

All the data are presented as mean \pm SD. One-way analysis of variance (ANOVA) was used in multiple group comparisons. Difference with $\mathrm{P}<0.05\left(^{(}\right)$or $\left.\mathrm{P}<0.01{ }^{(*}\right)$ was considered statistically significant. 


\section{Results And Discussion}

\section{Preparation and Characterization of Se@TP}

The light images and Tyndall effect of Se@TP were as shown in Fig. 1A, 1B and 1C, the results indicated that Se@TP nanoparticles were synthesized. The morphology of Se@TP presented spherical particles in Fig. 2A shape. TP modified with SeNPs formed more compact and stable globular nanocomposites. As shown in Fig. 2B, EDX indicated the signal of C (10\%) and O (8\%) that from TP, the percentage of Se atoms was $82 \%$. As shown in (Fig. 2C and 2D), Se@TP was decreased from $200 \mathrm{~nm}$ to $80 \mathrm{~nm}$ which indicated much smaller size. The zeta potential of SeNPs (-25 mv) was lower than Se@TP (8 mv), demonstrating the higher stability of Se@TP than SeNPs.

\section{Antiviral of Se@TP}

MDCK cells infected by H1N1 influenza was showed reduction in cells numbers and loss of cell-to-cell contract as shown in Fig. 3A. When co-treatment with Se@TP, the cells morphological were slightly changed. In Fig. 3B, the cell viability were 26\% (virus), 43\% (virus + TP), 67\% (virus + SeNPs) and 88\% (virus+Se@TP). The results indicated that the antiviral of SeNPs was effectively amplified by TP. Minimum inhibitory concentration (MIC) of drug A combination present in Se@TP of Se (125 $\mu \mathrm{M})$; MIC of drug B combination present in Se@TP of TP $(2.5 \mu \mathrm{M})$; MIC of drug A alone corresponded to free SeNPs $(1 \mathrm{mM}) ; \mathrm{MIC}$ of drug B alone corresponded to free TP $(10 \mu \mathrm{M})$. Fractional Inhibitory Concentration (FIC) was calculated as (MIC drug A combination/MIC drug A alone) + (MIC drug B combination/ MIC drug B alone $)=125 \mu \mathrm{M} / 1 \mathrm{mM}+2.5 \mu \mathrm{M} / 10 \mu \mathrm{M}=0.375$. FIC was 0.375 , below 0.5 , indicating synergy. In this study, the FIC index was basically interpreted as follows: FIC < 0.5, synergy; FIC between 0.5 and 2 , indifference; FIC > 2, antagonism. The results suggest that Se@TP effectively inhibited the proliferation of H1N1 influenza virus.

\section{Intracellular localization of Se@TP}

The lysosomes was found and increased in a time-dependent manner in MDCK cells as showen in Fig. 4. After30 min, Se@TP escapes from lysosomes and transported in the cytosol. Then distributed into the cells after $60 \mathrm{~min}$. This result showed the target organelle of Se@TP was lysosome.

\section{Detection of Caspase-3 activity}

The caspase-3 activities were 451\% (virus), 332\% (virus + TP), 290\% (virus + SeNPs) and 190\% (virus + Se@TP) as shown in Fig. 5. The treatment of H1N1 influenza virus infected MDCK cells remarkably increased the activity. TP and SeNPs slightly inhibited the caspase-3 activity, Se@TP significantly decreased the caspase-3 activity. The result showed that the Se@TP inhibits the H1N1 influenza virus through capase 3 activity.

\section{Inhibition of H1N1 influenza virus infection}


Typical apoptotic features with H1N1 influenza virus with nuclear condensation (blue fluorescence) and DNA fragmentation (green fluorescence) was exhibited by MDCK cells in Fig. 6. After treatment with $\mathrm{H} 1 \mathrm{~N} 1$ virus for $24 \mathrm{~h}$, the number of TUNEL-positive cells was significant increased. Co-treatment with Se@TP obviously inhibited the H1N1 influenza virus-induced changes. The results indicated that Se@TP inhibited the apoptosis of MDCK cells by H1N1 influenza virus.

\section{Inhibition of ROS generation by Se@TP}

Microvilli and mitochondria were observed in MCK cells. As shown in Fig. 7A When MDCK cells was incubated with H1N1 influenza virus, TEM image indicates distorted organelles, shrinking cytoplasm and condensed chromatin. The mitochondria of MDCK cells recovered the shape after treatment with Se@TP in Fig. 7B. The ROS generation was conducted by DCF determination to indicate the action mechanisms of Se@TP. The intracellular ROS generation were 430\% (virus), 332\% (virus + TP), 260\% (virus + SeNPs) and 130\% (virus + Se@TP) as shown in Fig. 8A. TP and SeNPs slightly inhibited the ROS generation. However, Se@TP remarkably decreased the ratio of ROS generation. The fluorescent intensity of DCF treated with H1N1 influenza virus was much stronger than TP, SeNPs and Se@TP in Fig. 8B. The results indicate the ROS participated in the antiviral action.

\section{In vivo antiviral}

Mice were infected with H1N1 virus and treated with TP, SeNPs and Se@TP, followed by HE staining, tunel analyses and immunohistochemical test of lungs tissues after being executed (Fig. 9A). As the HE staining present (Fig. 9B), H1N1 infected group manifested as alveolar collapse, perivascular and peribronchiolar edema. When treated with TP or SeNPs, the symptoms lessened. Se@TP attenuated the histopathological manifestations substantially. The result indicated that Se@TP protected the lungs from being injured. Se@TP nanoparticles prevent the DNA damage during H1N1 infection. Meanwhile, the HE stain and tunel analysis illustrated that Se@TP inhibited MDCK cells infection by H1N1 influenza virus. Caspase-3, P-AKT, T-AKT, P-P53 and P53 proteins were detected and positive after H1 N1 virus infection as showed in Fig. 10. The results demonstrated that Se@TP inhibited H1N1 influenza virus-induced MDCK cell apoptosis by ROS mediated AKT and p53 signalling pathway (Fig. 11).

\section{Conclusions}

In conclusion, Se@TP with lower toxicity exhibits superior antiviral abilities to prevent H1N1 influenza virus infection in this study. The mechanisms of antiviral showed that Se@TP inhibited caspase-3 mediated apoptosis through ROS generation. In vivo antiviral result showed that Se@TP inhibited MDCK cells apoptosis through regulating the AKT and p53 signalling pathways. In summary, the nanosystem of Se@TP might provide a promising selenium species with antiviral activity to against H1N1 influenza virus.

\section{Abbreviations}


FBS: Fetal bovine serum; DMEM: Dulbecco's modified eagle medium; MTT: Thiazolyl blue tetrazolium bromide; p-p53: phosphorylated p53; p-AKT: Phosphorylated AKT.

\section{Declarations}

Availability of Data and Materials

All data are fully available without restriction.

Authors' contributions

Changbing Wang, Mingqi Zhao and Zhengfang Lin designed the study, analyzed the experimental data and drafted the manuscript. Min Guo, Tiantian Xu and Ying Tang carried out the experiments. Yi Chen, Liang Hua, Jiayu Zhong, Tao Lin, Guangwan Lian and Huanhui Chen participated in the design. Bing Zhu and Yinghua Li refined the manuscript and coordination. All authors read and approved the final manuscript.

Acknowledgements

This work was supported by the Technology Planning Project of Guangzhou (201804010183), the Medical Scientific Research Foundation of Guangdong Province (A2018306), Pediatrics Institute Foundation of Guangzhou Women and Children's Medical Centre (YIP-2018-036 and YIP-2019-025), Pediatrics Institute Foundation of Guangzhou Women and Children's Medical Centre (IP-2018-004).The Guangzhou Medical University students' science and technology innovation project (2019AEK02 and2019AEK11).

Disclosure

The authors report no conflicts of interest in this work.

\section{References}

1. Dey P, Bergmann T, Cuellar-Camacho JL, et al. Multivalent Flexible Nanogels Exhibit Broad-Spectrum Antiviral Activity by Blocking Virus Entry. ACS Nano.2018;12 (7), 6429-6442.

2. Wang $C Z$, Han $H H$, Tang $X Y$, et al. Sialylglycan-Assembled Supra-Dots for Ratiometric Probing and Blocking of Human-Infecting Influenza Viruses. Acs Appl Mater Interfaces. 2017; 9(30):25164-25170.

3. Yang MY, Sunderland K, Mao CB. Virus-Derived Peptides for Clinical Applications. Chem Rev. 2017; 117(15):10377-10402.

4. Li YH, Lin ZF, Zhao MQ, et al. Silver Nanoparticle Based Codelivery of Oseltamivir to Inhibit the Activity of the H1N1 Influenza Virus through ROS-Mediated Signaling Pathways. Acs Appl Mater Interfaces. 2016; 8(37):24385-24393. 
5. Jung JH, Park BH, Oh SJ, et al. Integration of reverse transcriptase loop-mediated isothermal amplification with an immunochromatographic strip on a centrifugal microdevice for influenza A virus identification. Lab Chip. 2015;15(3):718-725.

6. Londrigan SL, Short KR, Ma J, et al. Infection of Mouse Macrophages by Seasonal Influenza Viruses Can Be Restricted at the Level of Virus Entry and at a Late Stage in the Virus Life Cycle. J Virol. 2015; 89(24):12319-12329.

7. Loveday EK, Diederich S, Pasick J, et al. Human microRNA-24 modulates highly pathogenic avianorigin H5N1 influenza A virus infection in A549 cells by targeting secretory pathway furin. J Gen Virol. 2015; 96:30-39.

8. Li YH, Lin ZF, Zhao MQ, et al. Reversal of H1N1 influenza virus-induced apoptosis by silver nanoparticles functionalized with amantadine. Rsc Adv. 2016; 6(92):89679-89686.

9. Liu YJ, Zhang LQ, Wei W, et al. Colorimetric detection of influenza A virus using antibodyfunctionalized gold nanoparticles. Analyst. 2015; 140(12):3989-3995.

10. Hai WF, Goda T, Takeuchi H, et al. Specific Recognition of Human Influenza Virus with PEDOT Bearing Sialic Acid-Terminated Trisaccharides. Acs Appl Mater Interfaces. 2017; 9(16):14162-14170.

11. Lin ZF, Li YH, Guo M, et al. The inhibition of H1N1 influenza virus-induced apoptosis by silver nanoparticles functionalized with zanamivir. Rsc Adv. 2017;7(2):742-750.

12. Kristensen AB, Lay WN, Ana-Sosa-Batiz F, et al. Antibody Responses with Fc-Mediated Functions after Vaccination of HIV-Infected Subjects with Trivalent Influenza Vaccine. J Virol. 2016; 90(12):5724-5734.

13. Li MK, Liu YY, Wei F, et al. Antiviral activity of $\beta$-Thujaplicin hydrochloride against herpes simplex virus I in vitro and in vivo. Int J Antimicrob Agents. 2018; 51(1):98-106.

14. Surov AO, Manin AN, Churakov AV, et al. New Solid Forms of the Antiviral Drug $\beta$-Thujaplicin: Crystal Structures, Thermodynamic Stability, and Solubility. Mol Pharm. 2015; 12(11):4154-4165.

15. Wang YT, Ding YW, Yang CG, et al. Inhibition of the infectivity and inflammatory response of influenza virus by $\beta$-Thujaplicin hydrochloride in vitro and in vivo (mice and ferret). Biomed Pharmacother. 2017; 91:393-401.

16. Kadam RU, Wilson IA. Structural basis of influenza virus fusion inhibition by the antiviral drug $\beta$ Thujaplicin. Proc Natl Acad Sci USA. 2017; 114(2):206-214.

17. Liu W, Li XL, Wong YS, et al. Selenium Nanoparticles as a Carrier of 5-Fluorouracil to Achieve Anticancer Synergism. ACS Nano. 2012; 6(8):6578-6591.

18. Li YH, Lin ZF, Xu TT, et al. Delivery of VP1 siRNA to inhibit the EV71 virus using functionalized silver nanoparticles through ROS-mediated signaling pathways. Rsc Adv. 2017; 7(3):1453-1463.

19. Gustafsson S, Mihranyan A. Strategies for Tailoring the Pore-Size Distribution of Virus Retention Filter Papers. Acs Appl Mater Interfaces. 2016; 8(22):13759-13767.

20. Tao WQ, Hurst BL, Shakya AK, et al. Consensus M2e peptide conjugated to gold nanoparticles confers protection against H1N1, H3N2 and H5N1 influenza A viruses. Antivir Res. 2017; 141:62-72. 
21. Vonnemann J, Sieben C, Wolff C, et al. Virus inhibition induced by polyvalent nanoparticles of different sizes. Nanoscale. 2014; 6(4):2353-2360.

22. Wang J, Zhu RR, Gao B, et al. The enhanced immune response of hepatitis $B$ virus DNA vaccine using SiO2@LDH nanoparticles as an adjuvant. Biomaterials 2014; 35(1):466-478.

23. Shen LH, Zhou J, Wang YX, et al. Efficient Encapsulation of Fe3O4 Nanoparticles into Genetically Engineered Hepatitis B Core Virus-Like Particles Through a Specific Interaction for Potential Bioapplications. Small. 2015; 11(9-10):1190-1196.

24. Zhang R, Lin Z, Lui VCH, et al. Silver nanoparticle treatment ameliorates biliary atresia syndrome in rhesus rotavirus inoculated mice. Nanomedicine.2017; 13(3):1041-1050.

25. Stevanovic M, Filipovic N, Djurdjevic J, et al. 45S5Bioglass (R)-based scaffolds coated with selenium nanoparticles or with poly(lactide-co-glycolide)/selenium particles: Processing, evaluation and antibacterial activity. Colloid Surface B. 2015; 132:208-215.

26. Huang YY, Luo Y, Zheng WJ, et al. Rational Design of Cancer-Targeted BSA Protein Nanoparticles as Radiosensitizer to Overcome Cancer Radioresistance. Acs Appl Mater Interfaces. 2014; 6(21):1921719228.

27. Jiang W, Fu Y, Yang F, et al. Gracilaria lemaneiformis polysaccharide as integrin-targeting surface decorator of selenium nanoparticles to achieve enhanced anticancer efficacy. ACS Appl Mater Interfaces. 2014; 6(16):13738-13748.

28. Cheng Z, Zhi X, Sun G, et al. Sodium selenite suppresses hepatitis B virus transcription and replication in human hepatoma cell lines. J Med Virol. 2016; 88(4):653-663.

29. Li YH, Guo M, Lin ZF, et al. Polyethylenimine-functionalized silver nanoparticle-based co-delivery of paclitaxel to induce HepG2 cell apoptosis. Int J Nanomedicine. 2016; 11:6693-6702.

30. Li YH, Lin ZF, Zhao MQ, et al. Multifunctional selenium nanoparticles as carriers of HSP70 siRNA to induce apoptosis of HepG2 cells. Int J Nanomedicine. 2016; 11:3065-3076.

31. Li YH, Lin ZF, Gong GM, et al. Inhibition of H1N1 influenza virus-induced apoptosis by selenium nanoparticles functionalized with arbidol through ROS-mediated signaling pathways. J Mater Chem B. $2019 ; 7: 4252-4262$.

32. Li YH, Lin ZF, Guo M, et al. Inhibition of H1N1 influenza virus-induced apoptosis by functionalized selenium nanoparticles with amantadine through ROS-mediated AKT signaling pathways. Int $\mathrm{J}$ Nanomedicine. 2018; 13:2005-2016.

33. Li YH, Li XL, Zheng WJ, et al. Functionalized selenium nanoparticles with nephroprotective activity, the important roles of ROS-mediated signaling pathways. J Mater Chem B. 2013;1(46):6365-6372.

34. Lin ZF, Li YH, Gong GF, et al. Restriction of H1N1 influenza virus infection by selenium nanoparticles loaded with ribavirin via resisting caspase-3 apoptotic pathways. Int J Nanomedicine. 2018; 13:57875797.

35. Guo M, Li YH, Lin ZF, et al. Surface decoration of selenium nanoparticles with curcumin induced HepG2 cell apoptosis through ROS mediated p53 and AKT signaling pathways. Rsc Adv. 2017;7(83):52456-52464. 


\section{Figures}
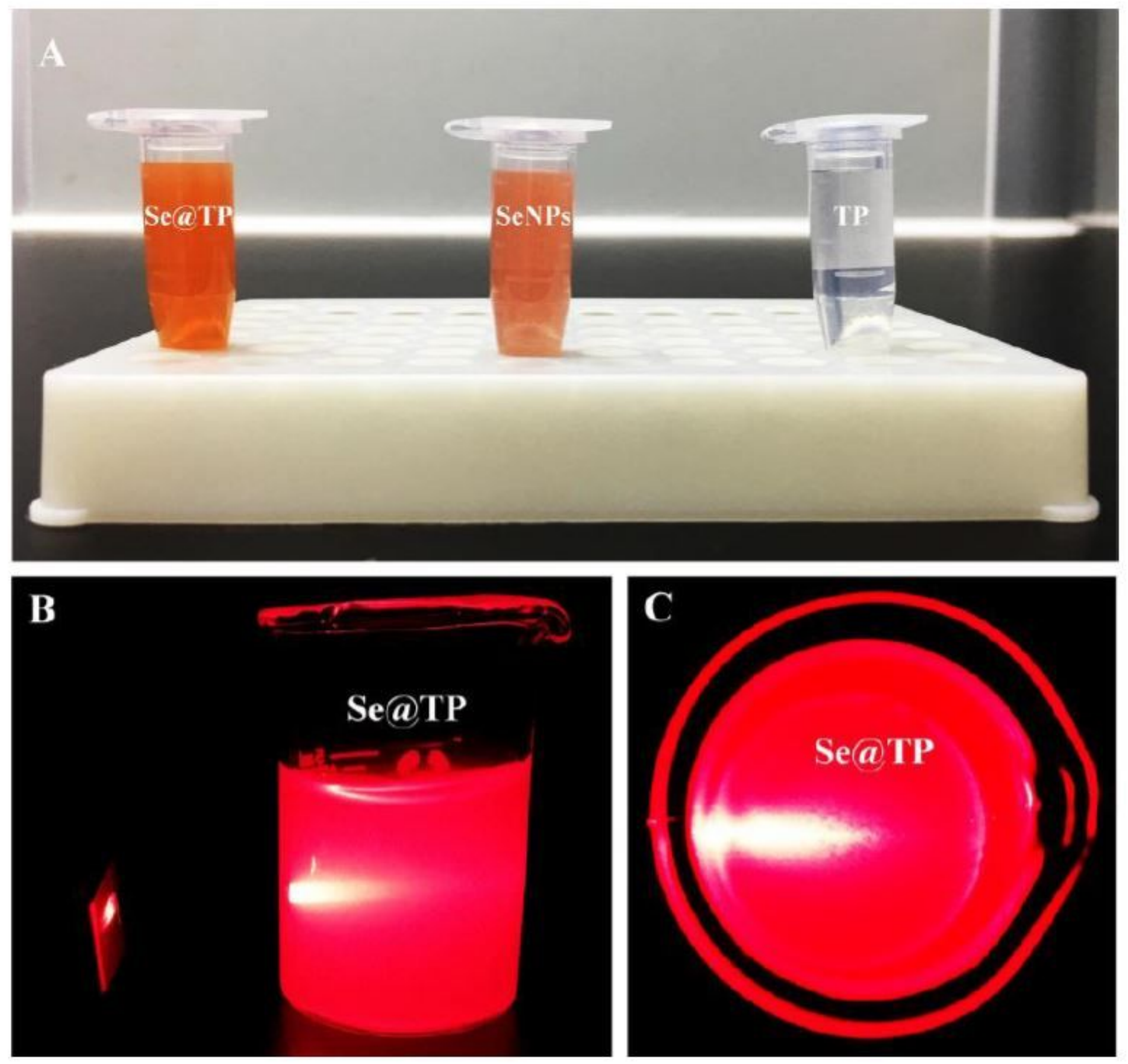

Figure 1

Light images of Se@TP, SeNPs and TP.(A) Color changes in Se@TP, SeNPs and TP. (B) and (C) Tyndall effect in Se@TP. 
A


B



C
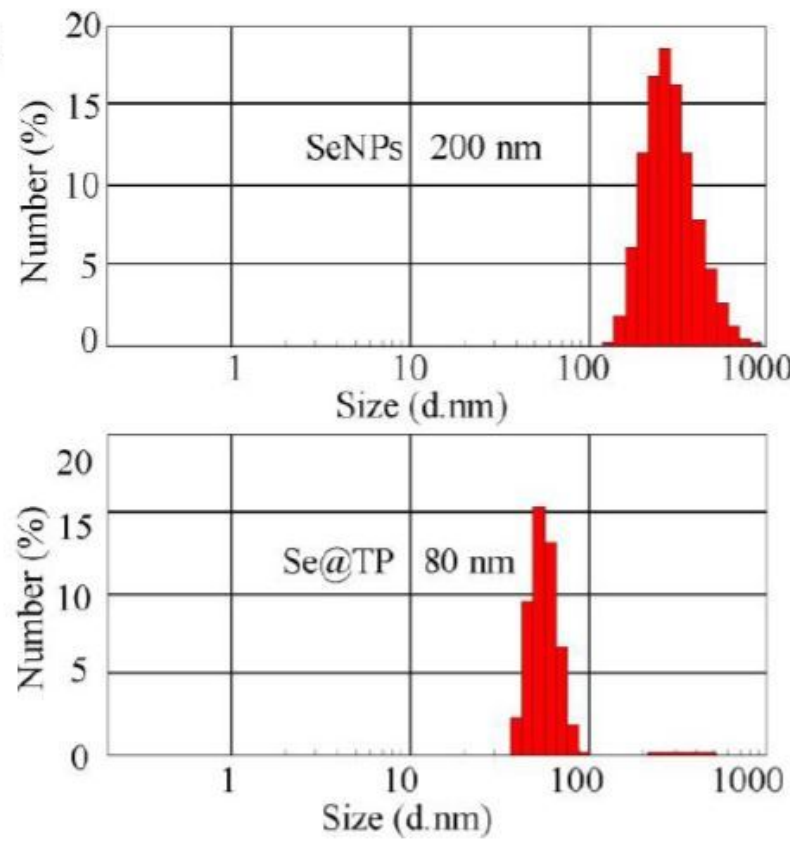
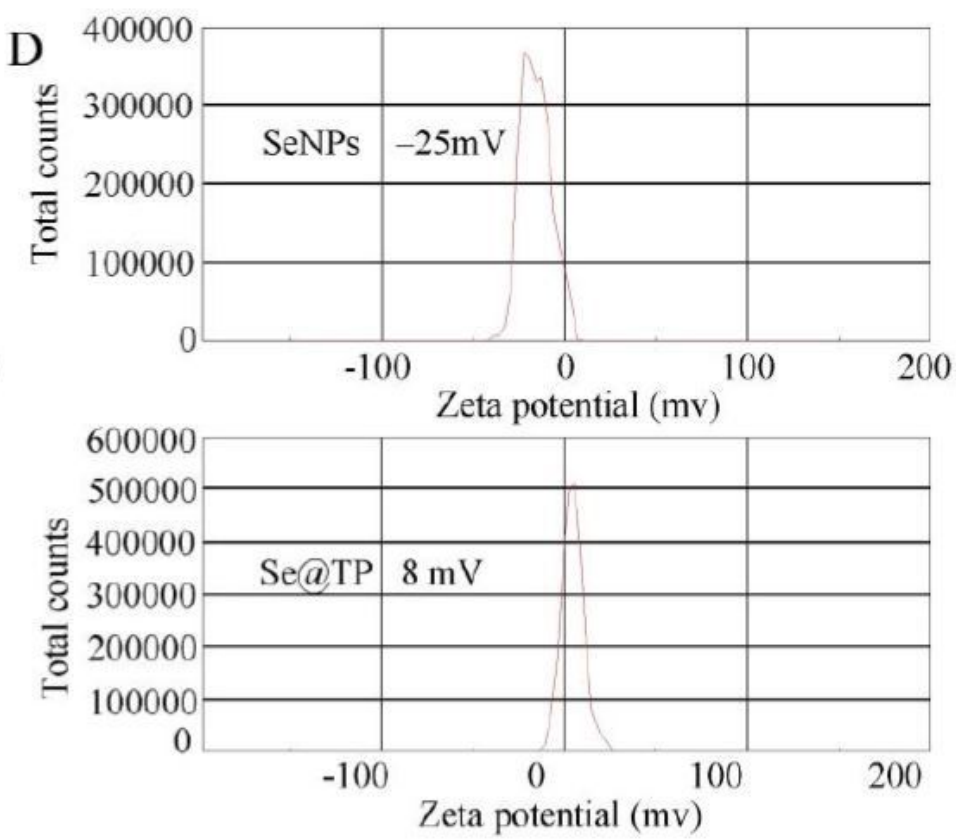

\section{Figure 2}

Characterization of SeNPs and Se@TP. (A) TEM images of SeNPs and Se@TP. (B) EDX analysis of Se@TP. (C) Size distribution of SeNPs and Se@TP. (D) Zeta potentials of SeNPs and Se@TP. 
A

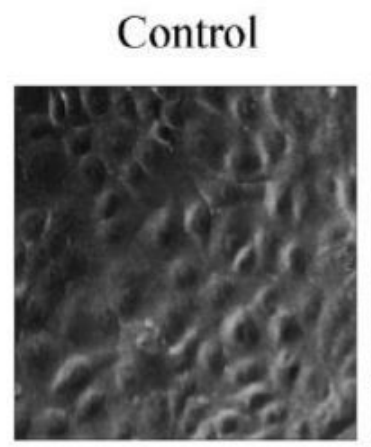

Virus $+\mathrm{TP}$

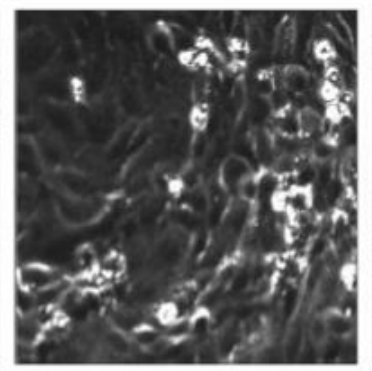

Virus + SeNPs



Virus+Se@TP

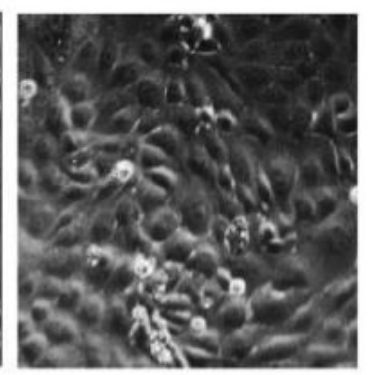

B

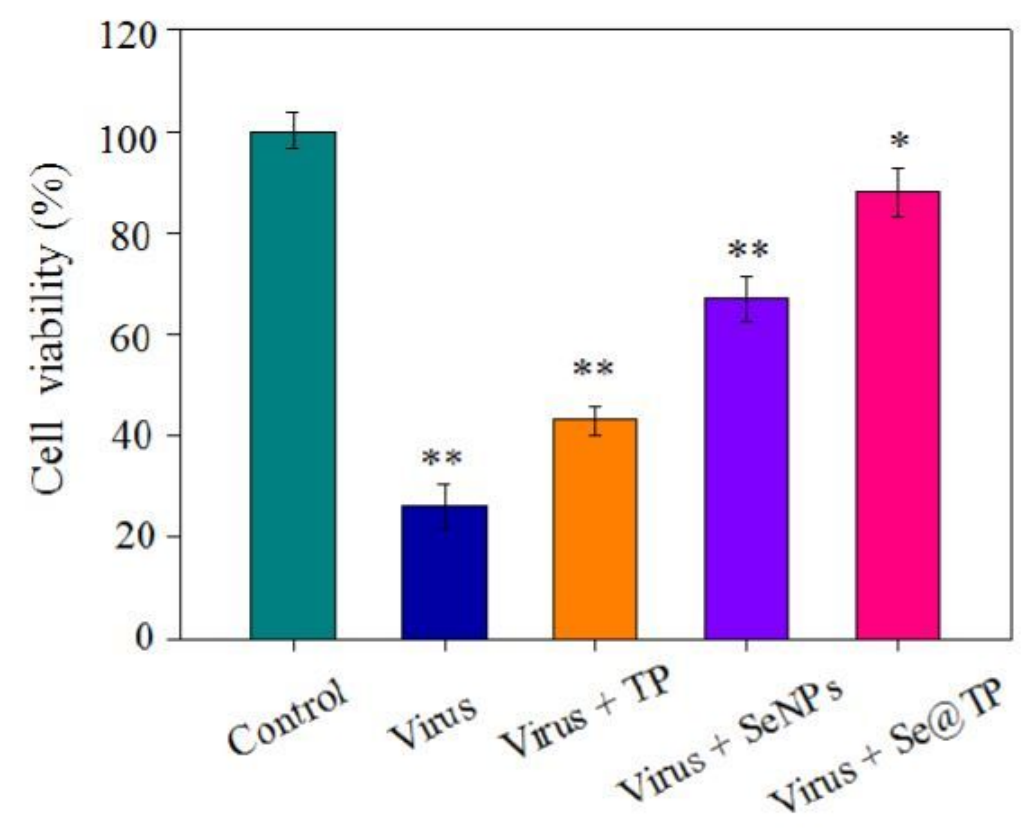

Figure 3

Effects of Se@TP on the growth of H1N1 infection of MDCK cells by MTT assay. (A) Morphological changes in H1N1-infected MDCK cells observed by phase-contrast microscopy. (B) Antiviral activity of Se@TP. Concentration of SeNPs was $1 \mathrm{mM}$ and TP $10 \mu \mathrm{M}$. Bars with different characters are statistically different at ${ }^{*} p<0.05$ or ${ }^{* *} p<0.01$ level. 


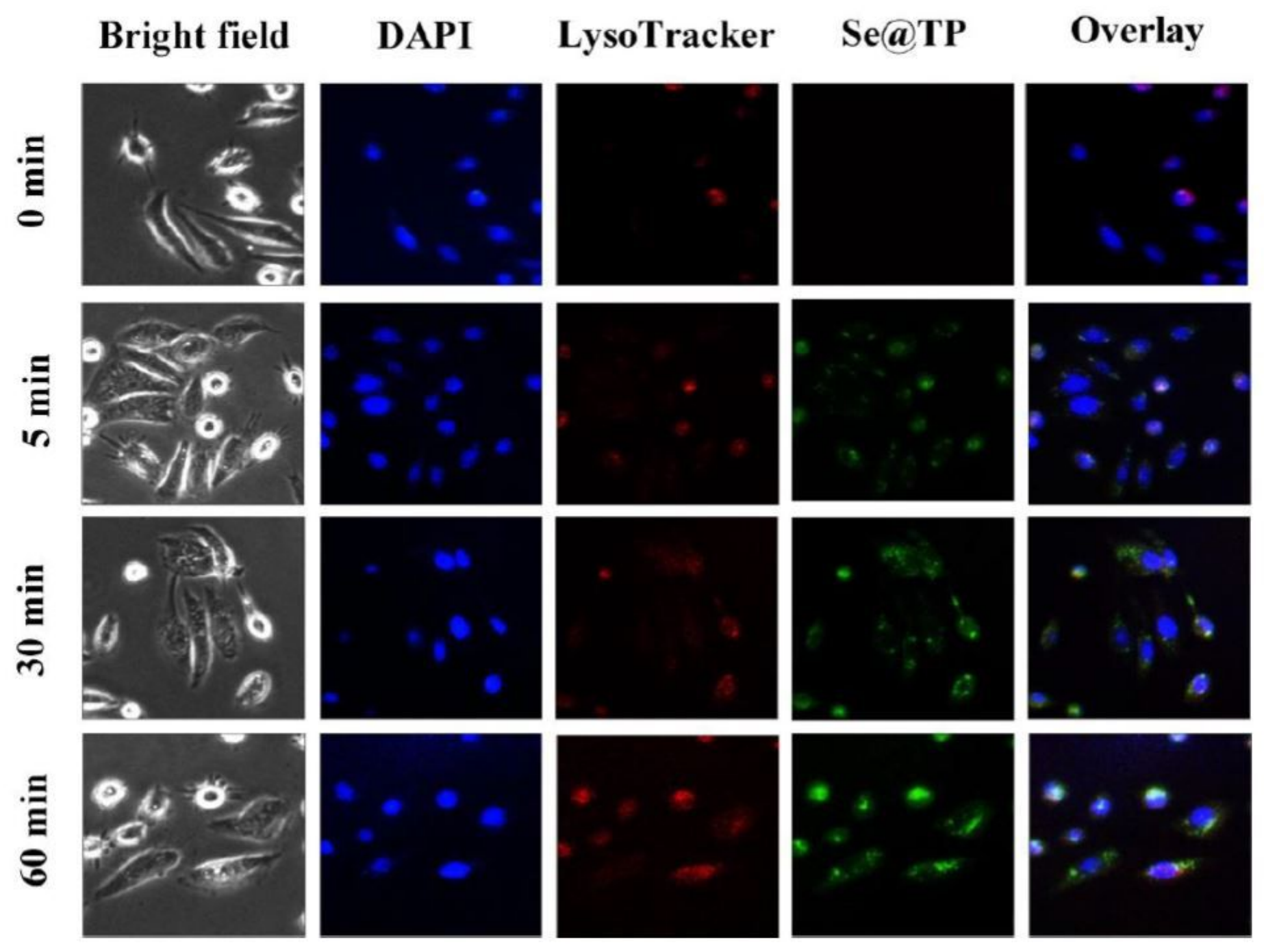

Figure 4

Localization of coumarin 6 loaded Se@TP in MDCK cells. The cells were treated with coumarin 6 loaded Se@TP for different periods of time and were observed under a fluorescent microscope s tained with lysotracker (red fluorescence, lysosome) and DAPI (blue fluorescence, nucleus) 


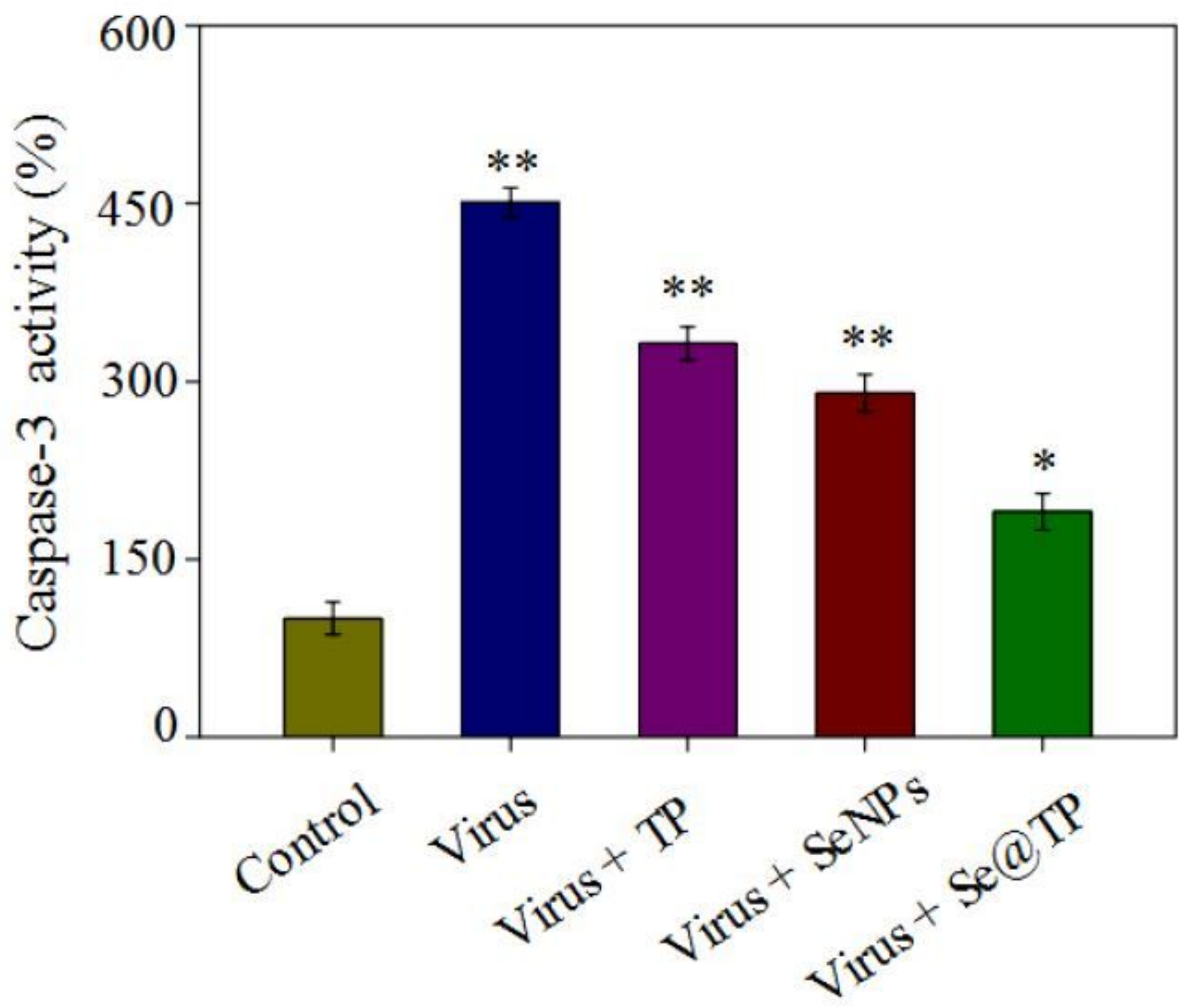

Figure 5

Inhibition of caspase 3 activity by Se@TP. Cells were treated with TP, SeNPs and Se@TP, and caspase 3 activity was detecte $d$ by synthetic fluorogentic substrate. Concentration of SeNPs was $1 \mathrm{mM}$ and TP 10 $\mu \mathrm{M}$. Bars with different characters are statistically different at $* p<0.05$ or ${ }^{*} \mathrm{p} p 0.01$ level . 


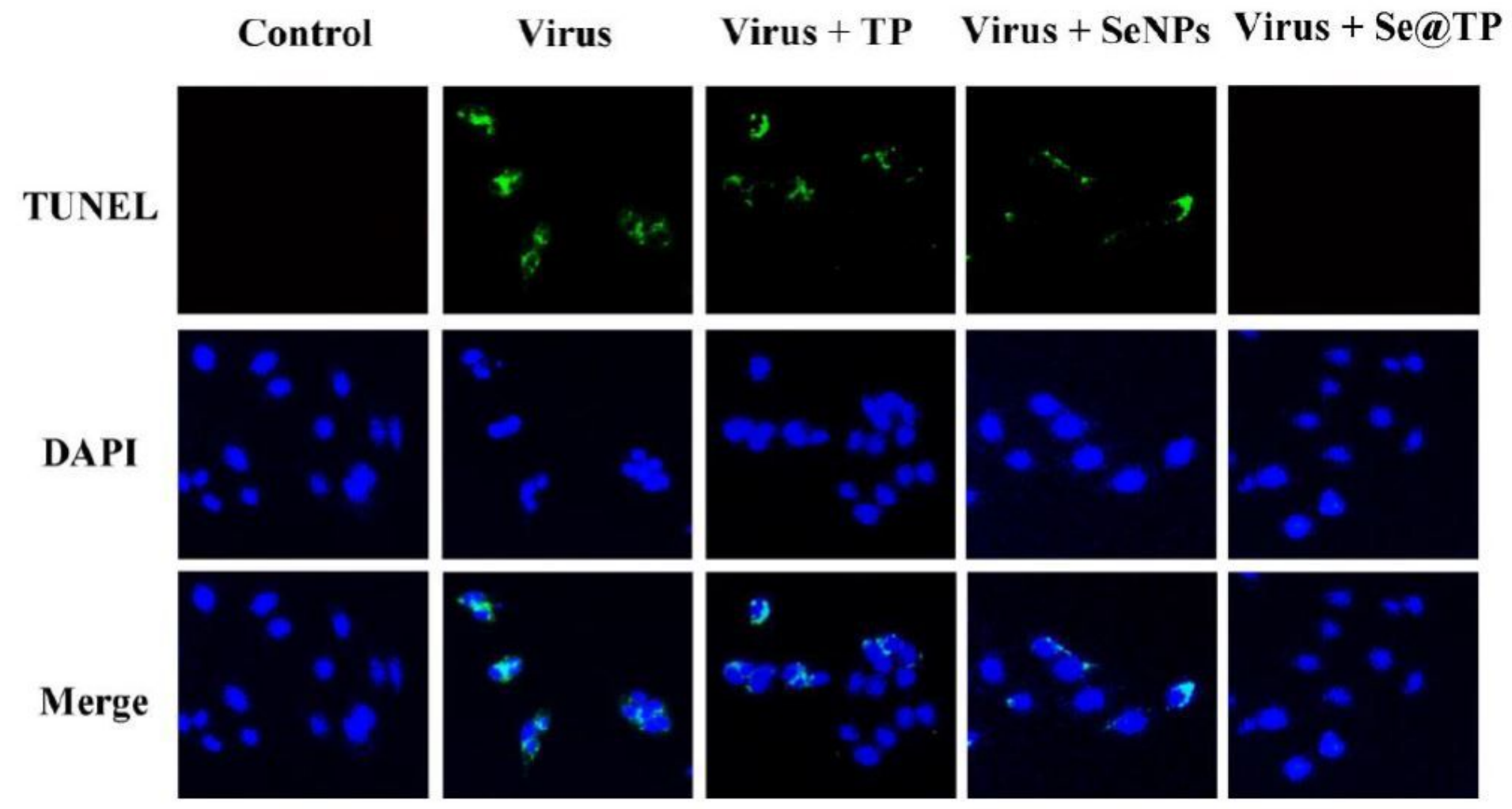

Figure 6

Se@TP induced apoptosis in H1N 1 infection of MDCK cells. DNA fragmentation and nuclear condensation as detected by TUNEL DAPI co staining assay. Concentration of SeNPs was $1 \mathrm{mM}$ and TP $10 \mu \mathrm{M}$. 


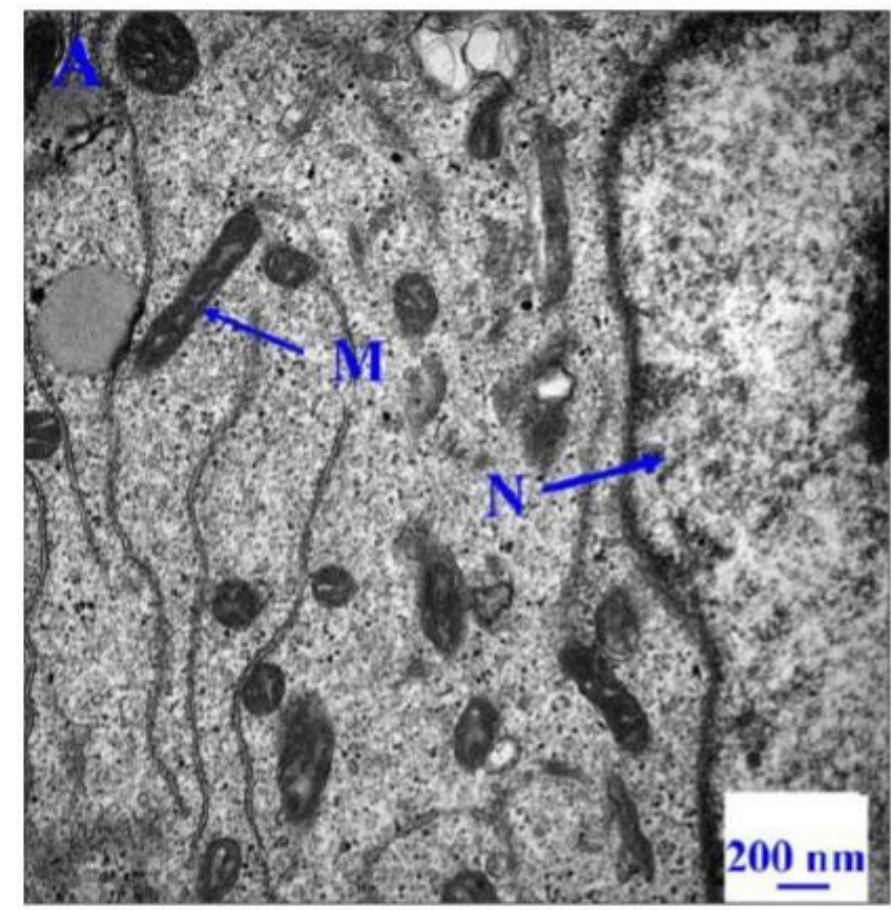

Virus

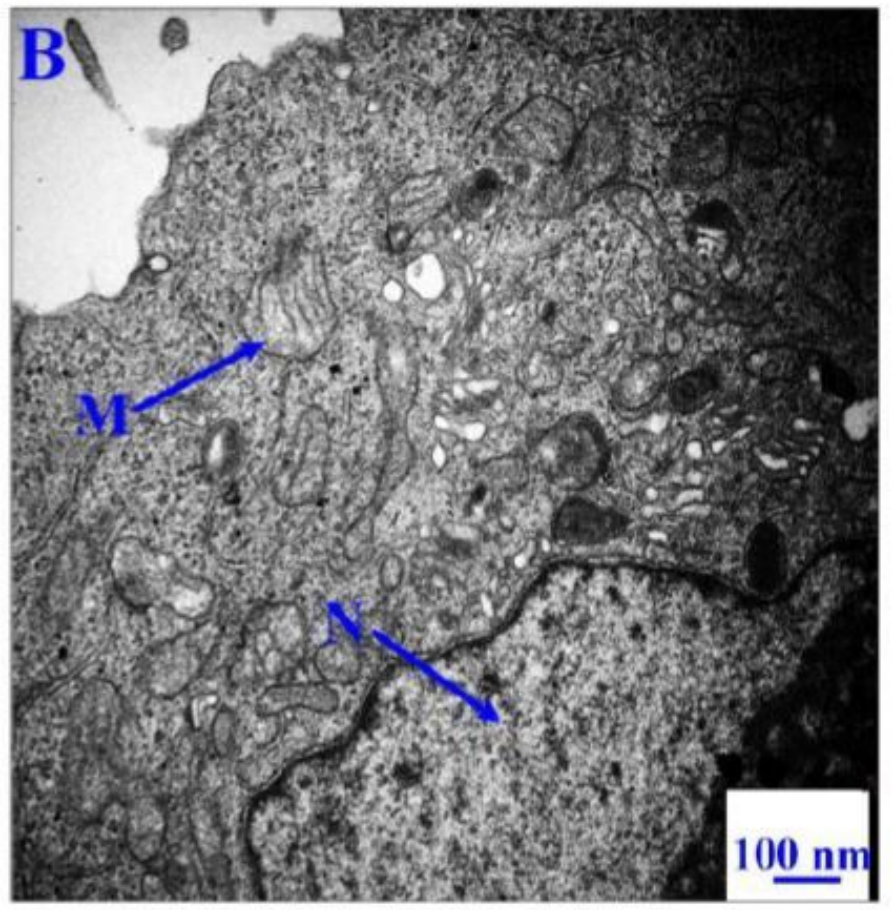

Virus+Se@TP

\section{Figure 7}

TEM images of thin sections of MDCK cells treate $d$ with Se@TP. (N: nucleus M: mitochondria Mv: microvillus) 
A

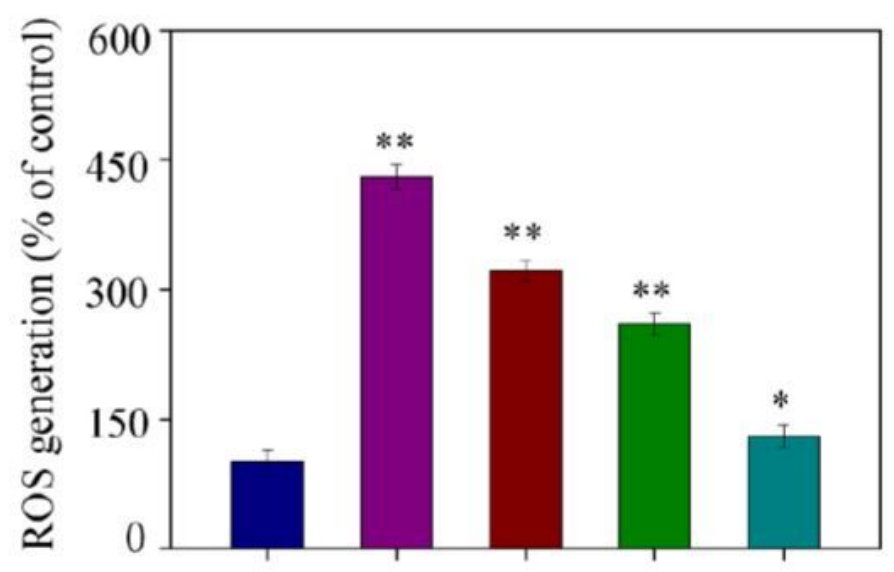

B

Control

Virus

Virus $+\mathrm{TP}$

Virus + SeNPs

Virus+Se@TP
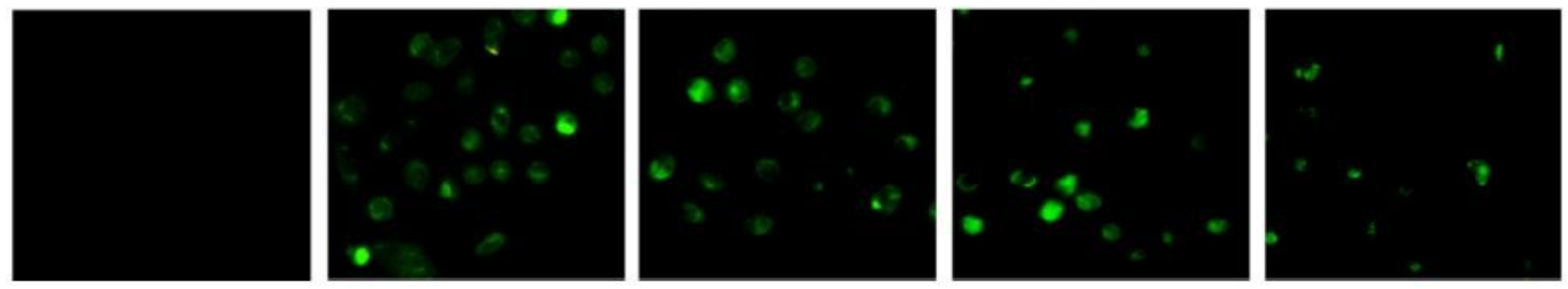

\section{Figure 8}

ROS overproduction induced by Se@TP in H1N1 infection of MDCK cells. (A) ROS levels were detected by DCF fluorescence intensity. (B) H 1N1 infection of MDCK cells preincubated with $10 \mu \mathrm{M} \mathrm{DCF}$ for 30 min and then treated with Se@TP. Concentration of SeNPs was 1 mM and TP $10 \mu \mathrm{M}$. Bars with different characters are statistically different at ${ }^{*} p<0.05$ or $* \star p<0.01$ level . 
A
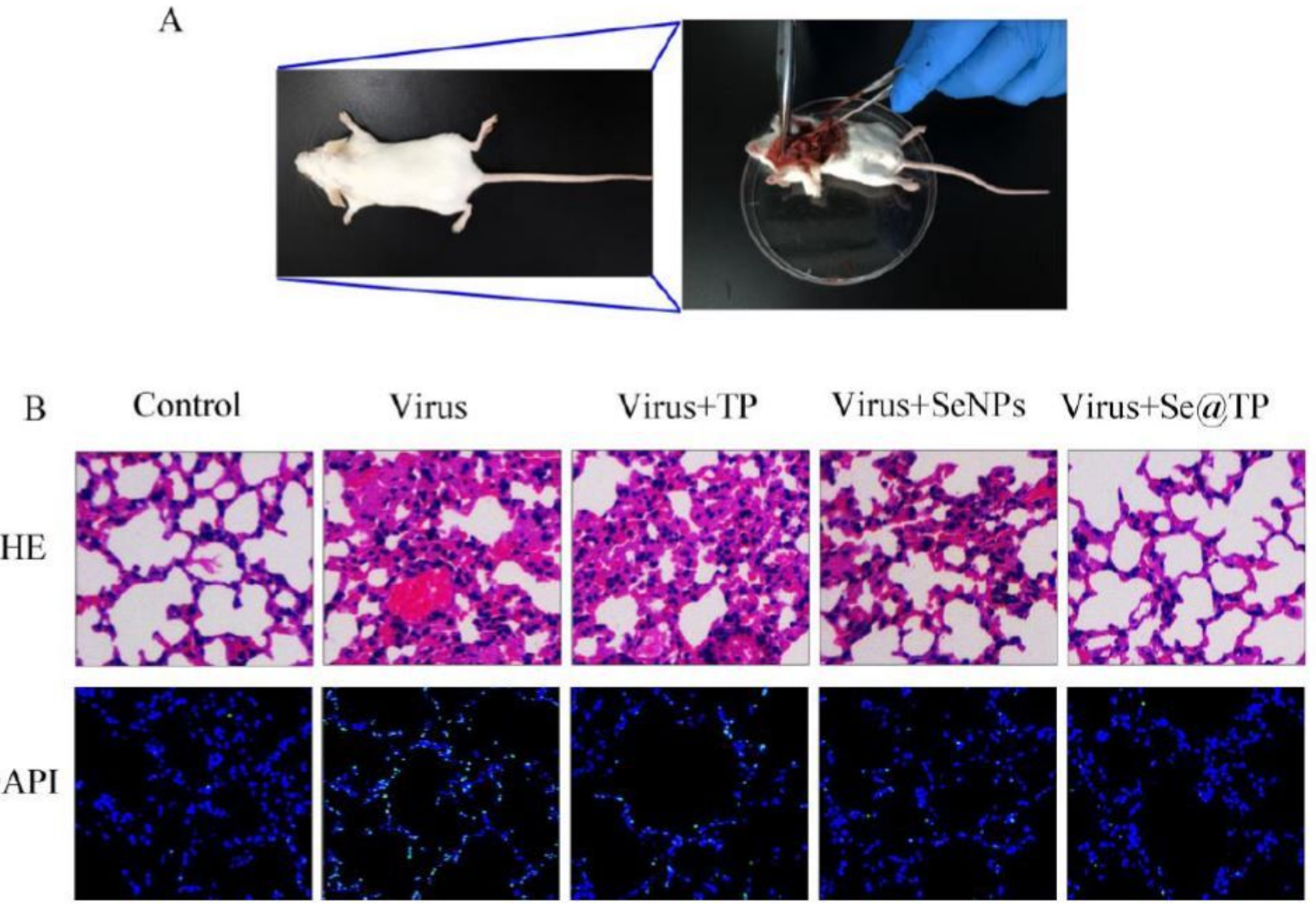

Figure 9

Istopathological analysis of Se@TP effects on the lung tissue sections of mice.(A) Photos of extra lung. (B) In vivo antiviral efficiency of Se@TP. Pathologic lesions of lung tissues of HE and TUNEL DAPI staining. 


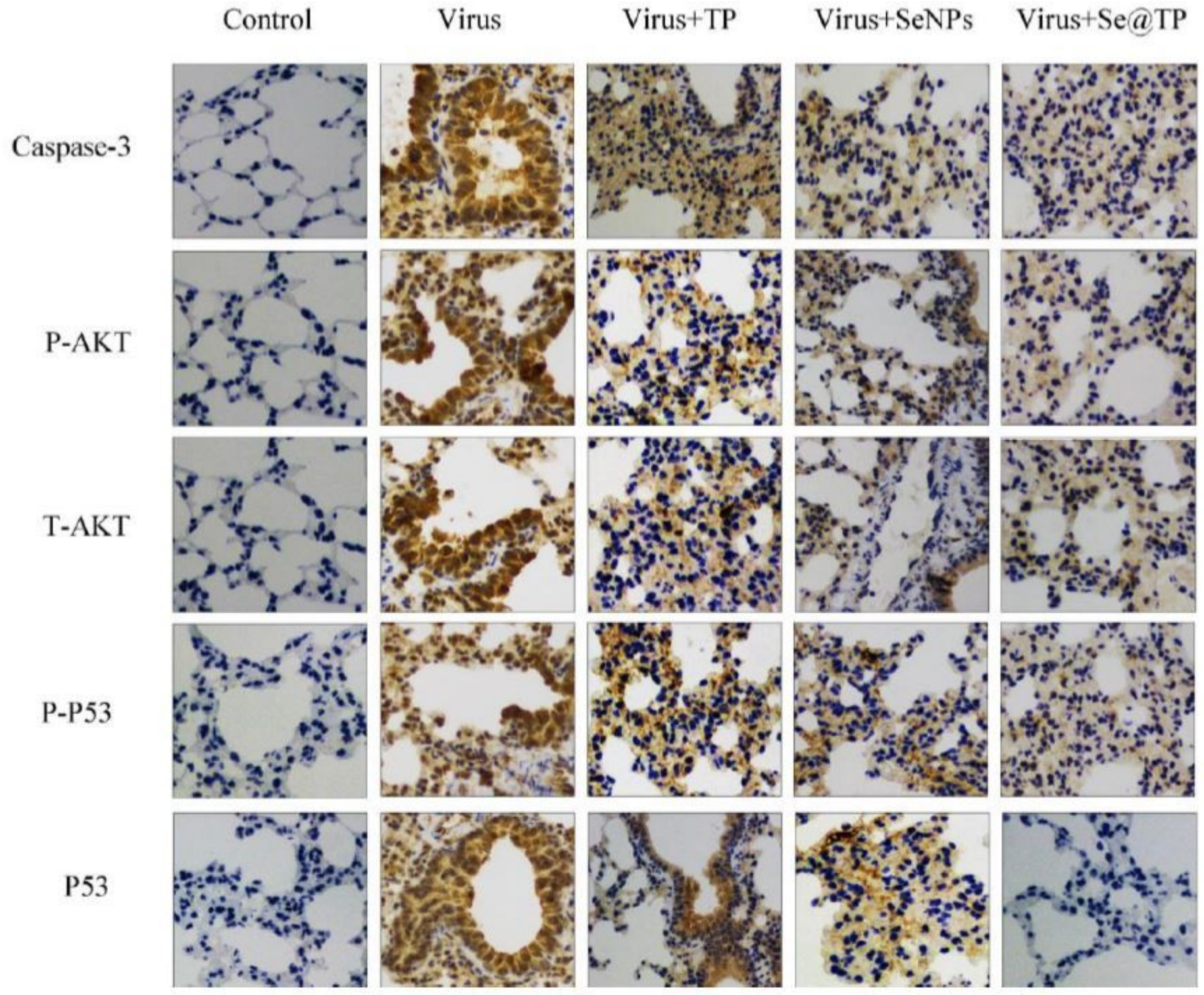

Figure 10

In vivo antiviral efficiency of Se@ TP by immunohistochemistry of. Mice without infection performed as control. 


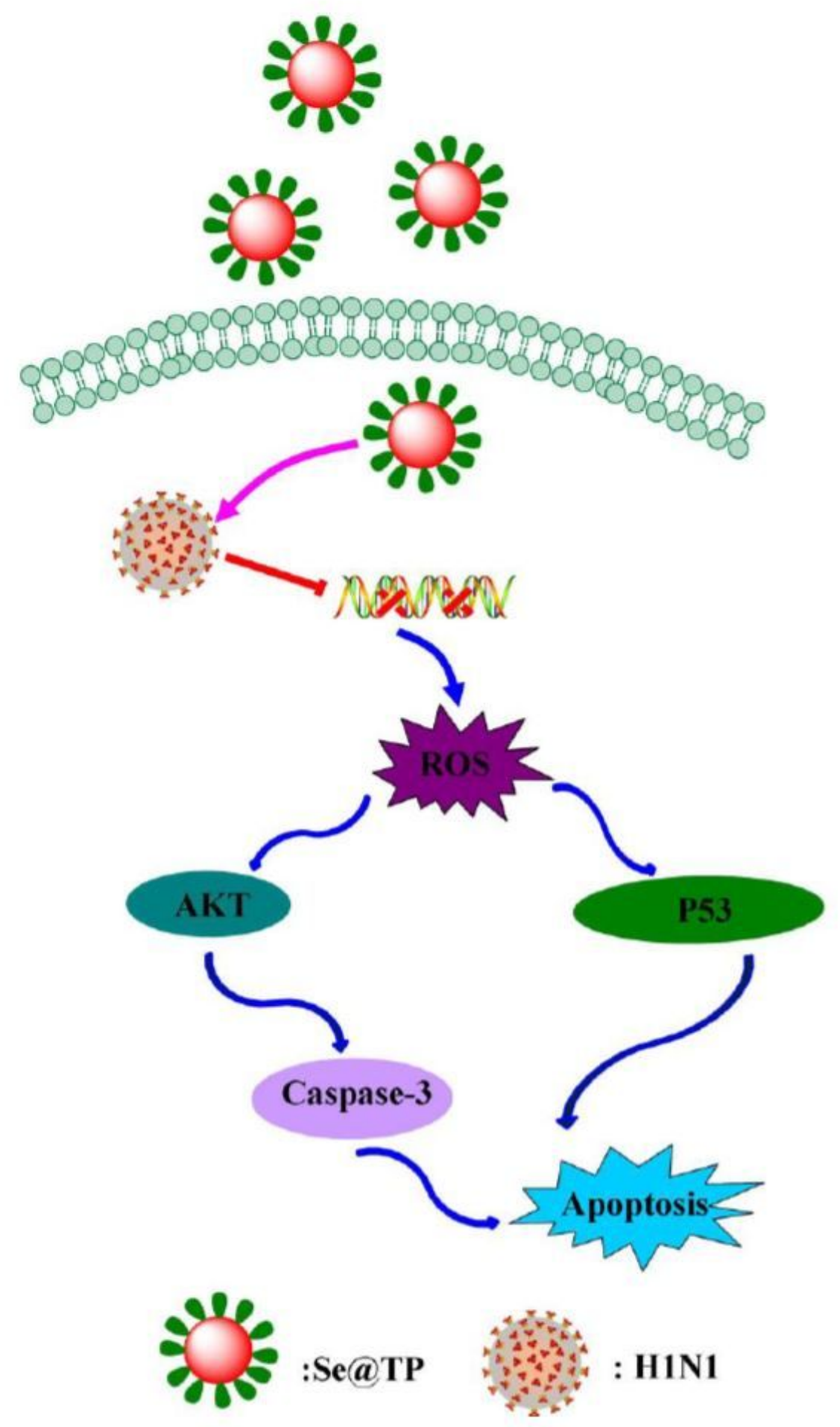

Figure 11

Apoptotic signaling pathways by TP in H1N1 infect ion of MDCK cells. The main signal ing pathway of ROS mediated AKT and P53 signaling pathways. 